\title{
Integración Curricular: respuesta al reto de educar en y desde la diversidad
}

\section{Curricular Integration: responding to the challenge of educating in and through diversity}

\section{Integração Curricular: resposta ao desafio de educar em e desde a diversidade}

\author{
Nuria Illán Romeu ${ }^{1}$ \\ Jesús Molina Saorín ${ }^{2}$
}

\begin{abstract}
RESUMEN
A través de este trabajo, presentamos nuestro modelo de diseño, desarrollo y evaluación de Unidades Didácticas Integradas (UDIs), describiendo todas y cada una de sus diferentes fases. Del mismo modo, exploramos sus potencialidades en la construcción de un currículo cimentado sobre los supuestos de la Integración Curricular. Se trata de un modelo que surge de un proceso de investigación llevado a cabo en diferentes centros educativos. Nuestro propósito último es, precisamente, tratar de llegar a un mayor número de profesores, maestros y educadores con el fin de que, tras su lectura, sean capaces de utilizarlo e implementarlo en diferentes escenarios.
\end{abstract}

Palabras-clave: integración curricular; diversidad educativa; unidad didáctica integrada.

${ }^{1}$ Doctora en Filosofía y Ciencias de la Educación. Profesora en la Universidad de Murcia (UMU), Murcia, España. nuriair@um.es <www.mvai.org>.

${ }^{2}$ Doctor Europeo en Pedagogía. Profesor en la Universidad de Murcia (UMU), Murcia, España. Profesor en la Universidad Nacional de Educación a Distancia (UNED), Madrid, España. jesusmol@um.es<www.um.es/jesusmolina>. 


\begin{abstract}
Through this work, we present our model of design, development and evaluation of Integrated Teaching Units (UDIs), describing each and every one of its different phases. Similarly, we explore their potential in building a curriculum founded on the assumptions of Curricular Integration. This is a model that emerges from a research conducted in different schools. Our ultimate purpose is, precisely, to try to reach a larger number of professors, teachers and educators so that, after reading, be able to use it and implement it in different contexts.
\end{abstract}

Keywords: curriculum integration; diversity education; integrated teaching unit.

\title{
RESUMO
}

Com este trabalho, apresentamos o nosso modelo de desenho, desenvolvimento e avaliação de Unidades Didáticas Integradas (UDIs), descrevendo todas e cada uma das suas diferentes fases. Da mesma forma, exploramos as suas pontencialidades na construção de um currículo construído sobre os supostos teóricos da Integração Curricular. Trata-se de um modelo nascido de um processo de pesquisa desenvolvido em vários centros educacionais. O nosso propósito último é, justamente, tentar atingir o maior número possível de professores, mestres e educadores, com a finalidade de que, após sua leitura, sejam capazes de usá-lo e aplicá-lo em diferentes cenários.

Palavras-chave: integração curricular; diversidade educativa; unidade didática integrada.

\section{Introducción}

En primer lugar, consideramos oportuno efectuar unas breves consideraciones sobre el origen y la trayectoria que ha tenido la investigación sobre Integración Curricular, la cual iniciamos junto a un grupo de maestros y maestras en la década de 1990. Es completamente justo reconocer a aquellos que, años atrás, no sólo tuvieron la creencia de que una escuela para todos era posible sino que, además, se pusieron a trabajar por conseguirla. En aquellos años, y a consecuencia de todo un trabajo previo de investigación llevado a cabo en cuatro centros de Educación Primaria de la Región de Murcia (España), iniciamos un proceso de diseño y puesta en marcha de una Unidad Didáctica Integrada (UDI), considerada como una estrategia para dar respuesta al difícil reto de atender a 
la diversidad del alumnado en un mismo tiempo y espacio. En aquel momento, este grupo de investigación inició una clara apuesta por encontrar soluciones a la atención a la diversidad en la etapa de educación secundaria, partiendo de toda una serie de elementos en torno a los cuales reflexionar y tratar de encontrar soluciones prácticas. Concretamente, deseamos destacar - a continuación - algunos de estos elementos:

\section{Elementos para la reflexión}

- La construcción de una escuela para todos pasa, necesariamente, por un profundo debate ideológico y por una toma de postura de todos y cada uno de sus implicados;

- Una educación comprensiva y atenta a la diversidad no es algo que pueda improvisarse;

- Una educación que quiera acoger a todos y cada uno de sus alumnos y alumnas ha de reconocer las diferencias individuales (no sólo las del alumnado, sino también del profesorado);

- El cambio hacia una escuela para todos crea unas condiciones para las que (prácticamente) nadie se encuentra preparado;

- Una educación atenta a la diversidad no puede seguir conviviendo con un tipo de organización escolar que invita al aislamiento del profesorado;

- La división del conocimiento en compartimentos estanco, con la consiguiente especialización del profesorado y separación de materias y áreas, choca frontalmente con la idea de promover un aprendizaje que proporcione claves para entender el mundo (en su globalidad) y no sólo una parte de él.

Por último, queremos mencionar todas y cada una de las acciones llevadas a cabo sobre este particular; unas reflejadas en informes de proyectos e investigación; otras recogidas en artículos y libros que hemos llevado a cabo juntos, compartiendo escenarios con maestros, maestras y toda una cohorte de alumnado. Gracias a todos ellos ha sido posible configurar lo que hemos venido denominando como el modelo de diseño, desarrollo y evaluación de Unidades Didácticas Integradas. Dicho modelo ha sido probado en diferentes escenarios pertenecientes al sistema de educación público y concertado, concretamente en las etapas de educación primaria y secundaria, obteniendo siempre grandes y esperanzadores resultados, a través de los cuales poder afirmar que la idea 
de una escuela para todos, atenta y respetuosa con las diferencias, que utiliza los contenidos como medios y no como fines en sí mismos, y preocupada por integrar el conocimiento es completamente posible.

\section{Qué hemos hecho y junto a quiénes hemos construido nuestro conocimiento}

Unos años antes de la década de 1990, iniciamos una firme y activa pertenencia al denominado Proyecto Atlántida: "Educación y cultura democrática". Se trataba de un proyecto nacional que perseguía, básicamente, rescatar la idea de aquel ser humano perdido (como lo fuera el continente - Atlántida), con todo el conjunto de valores democráticos aparejados a dicho concepto. En ese sentido, junto a un colectivo de profesores de educación secundaria de un centro denominado "Monte-azahar", diseñamos y pusimos en marcha nuestra primera Unidad Didáctica Integrada, la cual denominamos "Viaje con nosotros", y se llevó a cabo con un grupo de alumnos que finalizaba su escolaridad obligatoria (16 años). Este trabajo culminó con la presentación de la tesis doctoral del profesor Espinoza, que llevaba por título: "La construcción del proyecto curricular para el primer ciclo de educación secundaria obligatoria: una experiencia institucional". A este trabajo le siguió el esfuerzo colectivo de un equipo de profesores de educación secundaria pertenecientes al centro Monte-azahar, para quienes la Fundación Séneca (fundación de amparo a la investigación) concedió un proyecto de investigación denominado "Planificación, desarrollo y evaluación de unidades didácticas integradas para el primer y segundo ciclo de la ESO”. El trabajo desarrollado durante varios años culminó con la publicación de un libro titulado "La construcción del proyecto curricular en educación secundaria obligatoria. Opción integradora ante una sociedad intercultural". Una vez iniciados los primeros años del nuevo milenio, formamos un nuevo equipo con docentes pertenecientes al colegio de educación primaria "Maestro José Castaño" y al instituto de educación secundaria "Floridablanca" (ambos de Murcia), con un nuevo proyecto de investigación concedido por dicha Fundación "La construcción de unidades didácticas integradas para el primer y segundo ciclo de la ESO en los centros de la región de Murcia". Uno de los resultados más inmediatos fue la tesis doctoral del profesor Molina, denominada "Estudio de caso sobre la viabilidad de la Integración Curricular como proceso para atender a la diversidad del alumnado del programa de diversificación curricular" (dedicada al análisis de la experiencia en secundaria), y la publicación de un nuevo libro: "El euro para todos, una experiencia pedagógica" (que recogía los resultados obtenidos 
en educación primaria). Seguidamente, también sale a la luz un documento denominado: "El euro: ¿algo más que un cambio de moneda?", formando parte del anteriormente citado Proyecto de Innovación Atlántida "Educación y Cultura democrática". Por último, hemos publicado nuestro último libro titulado "Educar para la diversidad en la escuela actual. Una experiencia práctica de integración curricular", recogiendo nuestra experiencia ulterior en el diseño, desarrollo y evaluación de UDIs en la etapa de educación secundaria.

\title{
Qué entendemos por Integración Curricular
}

\author{
Entendemos por Integración Curricular:
}

[...] una modalidad de diseño del currículo, fundamentado en la concurrencia/colaboración/interconexión de los contenidos de varias disciplinas, para abordar un aspecto de la cultura escolar, a través de un modelo de trabajo cooperativo de profesores que incide, a su vez, en la metodología, en la evaluación y en el clima general del centro (ILLÁN; PÉREZ, 1999, p. 20).

Así pues, la Integración Curricular aspira a conseguir que los profesores no se limiten a llevar a la práctica las propuestas de las editoriales, sino que contextualicen realmente el currículo prescrito por la administración e intervengan activamente en su diseño y organización.

Del mismo modo, se apela a comprender el currículo no como producto, sino como proceso, al tiempo que trata de facultar a los profesores para intervenir, no sólo en su plasmación en la práctica y, eventualmente, en su evaluación, sino también en la planificación y organización de la misma.

\section{Formas de integrar el currículo}

Tal y como señalan Torres (1994) y Molina e Illán (2008), existen diferentes modalidades de integrar el currículo, de entre las cuales recogemos - a continuación - alguna de ellas: 
- Integración Curricular relacionando varias disciplinas. Supone coordinar las programaciones de varias disciplinas afines;

- Integración Curricular a través de tópicos. Las áreas implicadas coordinan sus programaciones alrededor de un centro de interés (tema). A partir de ese momento, no existe relación jerárquica entre ellas, sino que los intereses quedan subordinados a la propia interacción;

- Integración Curricular a través de cuestiones de la vida cotidiana. Desarrollando temas transversales difícilmente abordables desde el tratamiento unipolar que ofrece una sola disciplina;

- Integración Curricular a través de temas seleccionados por el alumnado. Organizando los contenidos alrededor de aquellos temas que los propios alumnos han seleccionado;

- Integración Curricular a través de conceptos. Se utilizan en grupos de alumnos con edades avanzadas (final de la ESO, Bachillerato, etc.), pues requiere una cierta madurez y dominio del pensamiento abstracto asociado a los conceptos (modernidad, tecnología, espacio, marginación, etc.);

- Integración Curricular a través de bloques históricos y/o geográficos. Se organizan los contenidos y las áreas a partir de acontecimientos históricos, espacios geográficos o periodos de la humanidad (la cultura egipcia, el Mar Mediterráneo, Afganistan, el imperialismo español, etc.);

- Integración Curricular a través de culturas o instituciones. Se organiza el currículo tomando como referente algunos grupos humanos significativos o alguna institución, asociación, organismo o corporación creada por las personas (los árabes, las sectas, las asociaciones, las ONGs, las residencias para ancianos, las prisiones para menores, etc.);

- Integración auricular a través de grandes descubrimientos o inventos. Se utilizan los descubrimientos e inventos como ejes vertebradores del currículo (la energía solar, el teléfono móvil, la electricidad, el autogiro, la vacuna, la pasteurización, el ordenador, Internet, etc.).

\section{Argumentos a favor de la Integración Curricular}

Desde nuestra experiencia en el desarrollo y puesta en marcha de la Integración Curricular, entendida como una estrategia capaz de contribuir a hacer 
realidad la educación inclusiva, destacamos los siguientes argumentos a favor de la Integración Curricular:

- Antídoto eficaz contra la cada vez más creciente fragmentación y especialización del conocimiento;

- Dota de significatividad y funcionalidad a los aprendizajes;

- Posibilita la adecuada sistematización de procedimientos comunes a todas aquellas disciplinas que no son objeto habitual de enseñanza explícita. Trabajar en proyectos integrados facilita que, por ejemplo, esa serie de verbos tan útiles para cumplimentar programaciones (como observar, medir, clasificar, comparar, comunicar, etc.), puedan llenarse de contenido real para los alumnos;

- Puede constituirse en un instrumento valioso para hacer frente al deterioro de la calidad académica en los centros escolares (sobrecarga; fragmentación y relevancia de los contenidos; tiempo y horarios restrictivos; reticencias de los profesores a utilizar prácticas innovadoras, etc.);

- Puede contribuir a aproximar la realidad circundante a las aulas, ofreciendo y capacitando a los estudiantes con unos aprendizajes que se consideran más adecuados en la sociedad actual (RELAN; KIMPSTON, 1991);

- La Integración Curricular defiende una visión del aprendizaje y la enseñanza como un camino que profesores y alumnos deben seguir para construir conexiones significativas entre el mundo del aula y el mundo real más amplio. El objetivo es que por la vía de la Integración Curricular "los alumnos comprenderán las relaciones entre cuerpos de conocimiento aparentemente dispares y apreciarán mejor la creciente complejidad del mundo en el que viven" (MARTÍN-KNIEP; FEIGE; SOODAK, 1995).

\section{Características de la Unidad Didáctica Integrada - UDI}

A partir de lo dicho hasta ahora, estamos en condiciones de adelantar cuáles son las características que definen a una Unidad Didáctica Integrada:

- Aborda un tópico o tema motivante para el alumnado;

- En el debate y selección del tópico intervienen profesorado y alumnado conjuntamente; 
- Tiene una duración definida y establecida previamente (dos o tres semanas);

- Persigue la consecución de competencias, objetivos generales y también didácticos;

- Aborda contenidos propios de la programación general de todas las áreas implicadas;

- Implica a todos los profesores de un grupo-clase;

- Atiende a la diversidad educativa desde el propio currículo, sin necesidad de adaptaciones ulteriores;

- Capacita al alumnado en la adquisición de conocimientos, habilidades y destrezas;

- Permite a los alumnos participar del proceso de toma de decisiones;

- El alumnado adopta un papel activo durante su proceso de aprendizaje, implicándose con la realidad que le rodea e interesa;

- Permite que alumnos con diferentes intereses, capacidades, ritmos de aprendizaje, etc., puedan participar simultáneamente en un mismo proceso educativo, intercambiando y compartiendo valores democráticos;

- Permite abordar temáticas en las que el currículo ordinario no suele detenerse, habitualmente, debido a su marcado carácter disciplinar;

- Conlleva la adopción y respeto por una serie de valores democráticos previamente consensuados en grupo y aceptados socialmente;

- Comprueba la significatividad del aprendizaje escolar en un entorno real y vital para el alumnado, en el cual han de poner en práctica aquello que han aprendido en el interior del centro;

- Implica la transferencia del conocimiento aprendido más allá del perímetro del centro;

- Permite trabajar no sólo contenidos conceptuales, sino también actitudinales y procedimentales;

- Concibe la enseñanza y el aprendizaje como un proceso dinámico, no como un producto controlable;

- Supone la colaboración e implicación conjunta entre profesorado, alumnado y familia;

- Supone un elemento regulador de la actividad de los docentes;

- Permite, con cierta facilidad, relacionar contenidos diferentes que de otro modo sería difícil vincular;

- Rescata el proceso de aprendizaje natural, alejado de divisiones artificiosas entre unas ciencias y otras;

- Implica a los alumnos en la construcción y reconstrucción activa de su propio proceso de aprendizaje y crecimiento. 
Fases del modelo de diseño, desarrollo y evaluación de Unidades Didácticas Integradas

\section{Fase I.: Formación y trabajo colaborativo del profesorado en tor- no al diseño de la Unidad Didáctica Integrada}

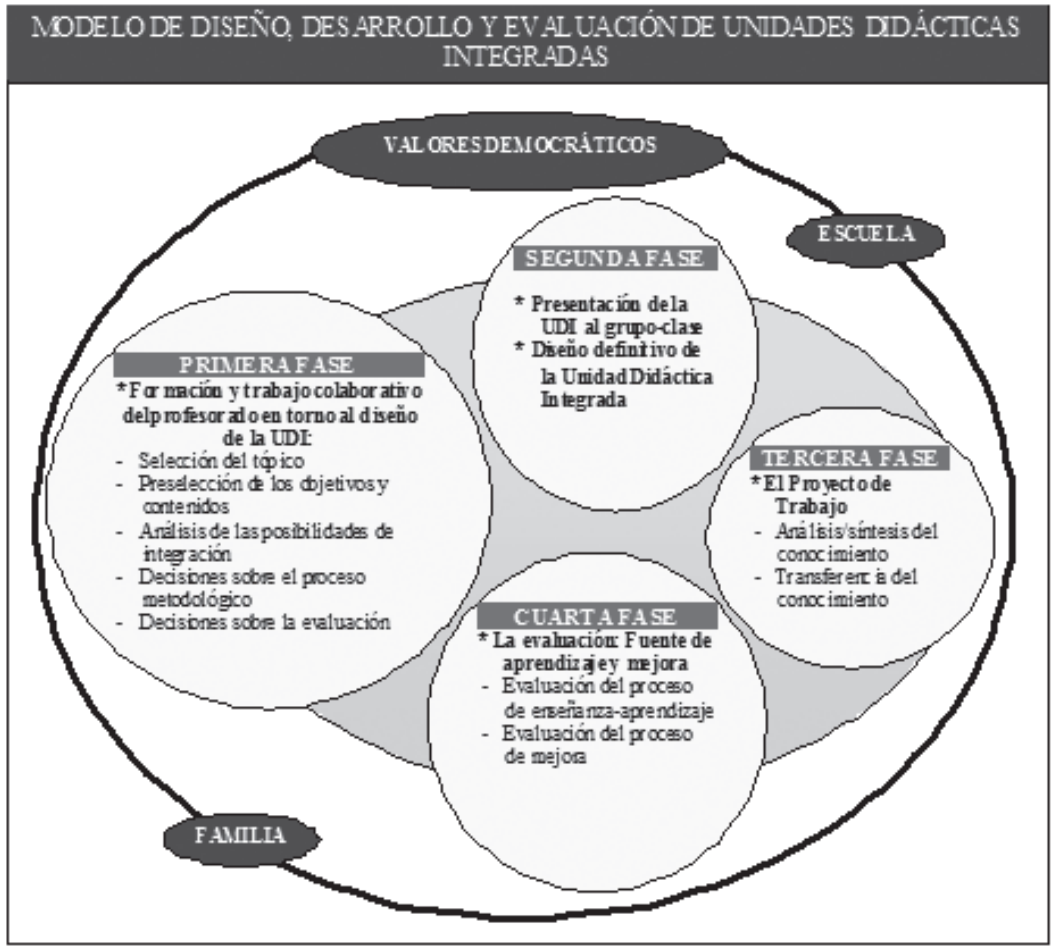

\section{La selección del tópico}

La selección del tópico representa algo más que la simple elección de un tema en torno al cual habrán de confluir distintos ámbitos del conocimiento. Es una magnífica oportunidad para dar respuesta a las necesidades e intereses de los alumnos, para abordar lo que realmente saben y desean saber de un tema determinado. Es una tarea que compromete tanto a profesores como alumnos, y que debe ser negociada con objeto de recoger opiniones, demandas concretas y permitir el establecimiento de acuerdos. 


\section{La preselección de los objetivos y contenidos}

Se trata de un proceso dinámico sometido a continua revisión y análisis. No se trata de un proceso lineal y aséptico - basado únicamente en los documentos oficiales -, sino que es permeable a toda una serie de influencias (escolares-familiares-culturales) en un espacio y tiempo determinado. Qué duda cabe, esta disponibilidad al cambio marca una gran diferencia respecto al modo tradicional de diseñar el currículo, donde siempre ha sido mayor el control que se ejerce sobre la procedencia del conocimiento, en detrimento de un ejercicio profesional creativo y autónomo. Precisamente esta apertura y permeabilidad también hace acto de presencia en el interior de la Unidad Didáctica Integrada. Así, cuando se preselecciona un determinado contenido, no sólo se tiene en cuenta el tópico y los objetivos a los que responde, sino que se deja espacio para la consideración de todas aquellas aportaciones que puedan enriquecer el aprendizaje. En ese momento, se abre una vía de doble dirección en la que el contenido seleccionado tratará de materializarse en una determinada actividad, tratando de encontrar la necesaria significatividad a la hora de ponerse en relación con otras actividades.

\section{Análisis de las posibilidades de integración}

Efectuada esta preselección, el grupo abre paso a una de las tareas más intensas del proceso de planificación: el análisis de las posibilidades de integración. Es aquí donde el trabajo colaborativo del profesorado encuentra su máxima expresión en el uso y manejo del currículo, y donde se marca la diferencia entre una unidad didáctica común y una Unidad Didáctica Integrada. Al finalizar dicho análisis, se dispone de un listado de los contenidos propios de la UDI. En este punto, la formulación de los contenidos integrados ha de ser capaz de expresar el tratamiento integrado del conocimiento. En definitiva, el enunciado de un determinado contenido integrado debe reflejar la interconexión entre aquellas áreas o disciplinas que habrán de desarrollarse a través de toda una serie de actividades, las cuales se interrelacionan, a su vez, con un determinado núcleo de contenido integrado.

Encontrar una estrategia capaz de reflejar todo este proceso y, al mismo tiempo, constituirse en un recurso didáctico capaz de guiar el proceso de enseñanza-aprendizaje, no resulta una tarea fácil. Nuestra experiencia en la continua inspección de las UDIs elaboradas (ILLÁN; PÉREZ, 1999; LOZANO; ILLÁN, 2001; MOLINA; ILLÁN, 2008), nos lleva a considerar y a seguir 
proponiendo el empleo de mapas conceptuales (Figura I). Se trata de mapas que se van construyendo a medida que emergen las aportaciones y reflexiones del grupo de profesores. Cada mapa recoge los distintos contenidos a trabajar en el transcurso de la unidad. A su vez, los contenidos aparecen organizados en torno a lo que hemos denominado como núcleos de contenido integrado. Por su parte, cada núcleo de contenido integrado agrupa, a su vez, a una serie de actividades integradas.

\section{Decisiones sobre el proceso metodológico}

Formando parte del proceso metodológico, hemos incluido los siguientes apartados:

a) Principios de intervención educativa;

b) Estructura organizativa del aula;

c) Colaboración familia-centro;

d) Evaluación.

a) Principios de intervención educativa. Nuestro modelo participa plenamente de los presupuestos teóricos en los que se sustenta el constructivismo, los cuales responden a los siguientes enunciados:

\section{Sin motivación no se construyen aprendizajes}

Si entendemos que la construcción del aprendizaje es una tarea que implica activamente al alumno, entonces resulta imprescindible que éste pueda encontrar sentido y motivación para realizar el esfuerzo que requiere dicha construcción (SOLÉ, 1991; ARNÁIZ; LOZANO, 1996). Por tanto, los aprendizajes que el niño realiza contribuirán a su desarrollo únicamente en la medida en que estos sean significativos, lo cual requiere que los alumnos tengan la oportunidad de establecer relaciones entre sus experiencias previas y los nuevos aprendizajes. En definitiva, el proceso que conduce a la realización de estos aprendizajes requiere que las actividades y tareas que se lleven a cabo tengan un sentido claro para los alumnos (MEC, 1992).

Es necesario relacionar las experiencias previas y los nuevos aprendizajes

La posibilidad de atribuir significado a los contenidos de aprendizaje remite a los conocimientos previos que el alumno ya posee y a la necesidad de 
establecer relaciones entre unos y otros (SOLÉ, 1991; COLL, 1987; ZABALA, 1989; ALMEIDA; GUISANDE; PRIMI; LEMOS, 2008). El conocimiento de las ideas o concepciones de los alumnos acerca de los contenidos escolares trabajados, así como las representaciones de estos sobre los contenidos objeto de aprendizaje, constituyen el primer paso de un proyecto integral de aprendizaje, al tiempo que resultan de suma importancia, tanto para mejorar la enseñanza de dichos contenidos como la práctica educativa en general.

Tal y como apunta Pozo (1989), el aprendizaje significativo es siempre el producto de la interacción entre un conocimiento previo activado y una información nueva. Entre las condiciones necesarias para lograr ese aprendizaje, un requisito esencial es disponer de técnicas y recursos que permitan activar los conocimientos previos de los alumnos, con objeto de confrontarlos con la nueva información. En esta línea, nosotros tomamos como punto de partida un tópico (como, por ejemplo, el euro), el cual suele resultar muy interesante tanto para alumnos, como también para el profesorado. Además, prestamos mucha atención a la exploración de los conocimientos previos de nuestros alumnos, como punto previo e ineludible a la hora de llevar a cabo la preselección de los objetivos, contenidos y analizar sus posibilidades de integración.

En este sentido, el proceso que llevamos a cabo en torno a los conocimientos previos, como parte esencial del diseño de la Unidad Didáctica Integrada, contribuye a que:

- Los alumnos adopten un papel más activo en el proceso de enseñanza-aprendizaje;

- Los profesores pueden incorporar dicho conocimiento a la hora de efectuar los ajustes y las acomodaciones necesarias, de modo que sean capaces de aminorar los posibles desfases (tanto por exceso como por defecto) entre lo que los alumnos ya conocían y lo que se proponía por conocer, determinando así la provisión de las ayudas que puedan facilitar su apropiación.

Tal y como apunta Solé (1991, p. 34):

[...] no hay que olvidar que cuanto mayor sea la distancia entre lo que los alumnos saben y lo que se les presenta, mayor esfuerzo se requerirá por parte de todos para que puedan establecer las relaciones necesarias para aprenderlo significativamente. 


\section{El aprendizaje, efecto y causa del desarrollo}

Entre el desarrollo de una persona y los aprendizajes que realiza, se establece una relación interactiva: el aprendizaje depende del grado de desarrollo, pero también posibilita un aumento del mismo, el cual, a su vez, permite hacer nuevos aprendizajes que abren nuevas vías de desarrollo (PUJOLÀS; RUIZ, 2000). Por lo tanto, todas las personas son educables y pueden aprender más de lo que han aprendido hasta un momento determinado (COLL, 1990). Durante el desarrollo de la Unidad Didáctica Integrada "El euro, ¿algo más que un cambio de moneda?", trabajamos mucho para modificar nuestra manera de situarnos ante los procesos de enseñanza-aprendizaje. Teníamos claro que si pretendíamos estimular los procesos cognitivos, debíamos diseñar y promover situaciones educativas en las que la observación y el descubrimiento que iban a realizar nuestros alumnos fuese retomado y elevado a un nivel de reflexión capaz de propiciar cambios, modificaciones y reestructuraciones en sus esquemas previos de actuación.

\section{Para construir conocimientos es necesaria la interacción social}

Si bien es cierto que es el alumno quien construye el conocimiento, relaciona, asimila e interpreta los datos del entorno, los profesores somos los encargados de crear las condiciones adecuadas para que dicho proceso pueda ser llevado a cabo. Por tanto, es el adulto quien aporta al niño situaciones significativas, hace que la actividad del niño sea autoestructurante o que, por el contrario, no tenga mayor impacto sobre el aprendizaje. Junto esto, a través de su estimulación (directa e individual), da respuestas las acciones del niño, las cuales pueden animarlo a seguir adelante o a desmotivarse ante una tarea determinada. Para Vygotsky (1979), el profesor no es sólo optimizador del desarrollo (por ser la persona que aporta situaciones didácticas estimulantes para el niño), sino que es un factor imprescindible. Lo que el niño hace hoy, con ayuda del adulto, es lo que mañana podrá hacer por sí solo. El área de desarrollo potencial es la que posibilita el desarrollo efectivo del niño. Según se formula en la tesis vygostskyana, la enseñanza adelanta al desarrollo desde el momento en que el educador crea el área de desarrollo potencial. Por tanto, sin la actuación del profesor el desarrollo efectivo no sería posible. Por todo lo dicho, hemos procurado que el conocimiento se fuera construyendo a través de un proceso de interacción entre los alumnos, el profesor y el contenido. Así, los procesos de enseñanza-aprendizaje desarrollados en el aula para la Unidad Didáctica Integrada han comportado el análisis de estos tres componentes de forma interrelacionada y no aislada. Estamos convencidos, y coincidimos con 
otros autores (GÓMEZ-GRANELL; COLL, 1994; COLL et al., 1992; MOLINA, 2009), que es necesario analizar no sólo la actividad constructiva de los alumnos y alumnas (ideas previas sobre el euro, predisposición y motivación para el aprendizaje del mismo, etc.), sino también los mecanismos de influencia o de ayuda pedagógica. Del mismo modo, coincidimos también con Coll (1990), Echeita y Martín (1990) y Pujolàs y Ruiz (2000), en que la ayuda del profesor debe ser lo más ajustada posible a las características y necesidades de cada alumno, si con ello pretendemos que nuestros alumnos tengan la posibilidad de construir (o reconstruir) activamente sus propios conocimientos sobre la base de los que ya tiene adquiridos. En consonancia con estos planteamientos, hemos favorecido que, durante en el transcurso de la UDI:

- Los alumnos interactuasen para pedir o prestar ayuda;

- Sintieran la necesidad de organizar sus ideas, con el fin de que tomaran conciencia de lo que sabían realmente, e identificaran sus posibles errores o lagunas;

- Valores como el respeto, tolerancia, cooperación, etc., encontraron su vehículo de expresión natural en las distintas actividades desarrolladas en pequeño y gran grupo;

- Potenciar el trabajo cooperativo, con la finalidad de que los alumnos aprendiesen a tener en cuenta distintos puntos de vista, fueran capaces de incorporar (ante una misma propuesta de trabajo) distintos ritmos de aprendizaje y, por último, tener en cuenta la necesidad de ayuda que tienen algunos de sus compañeros.

b) Estructura organizativa del aula. Por lo que respecta a la organización del aula en la que llevamos a cabo la experimentación de las UDIs, decir que la clase solemos dividirla en varios grupos (de cuatro o cinco alumnos y buscando la mayor heterogeneidad posible). Los criterios aplicados para la formación de los grupos son:

- En cada grupo debe haber niños y niñas;

- Las capacidades de los alumnos deben ser diferentes (por exceso y por defecto);

- En la medida de lo posible, cada grupo estará compuesto por un alumno de rendimiento alto, dos alumnos de rendimiento medio y uno de un rendimiento bajo;

Junto al trabajo cooperativo en pequeño grupo, también diseñamos actividades para llevar a cabo en gran grupo (por ejemplo: qué sabemos del euro, qué queremos saber, presentación de nuevos contenidos, etc.). También es importante dejar claro en este punto que, si bien es verdad que la mayor parte de las actividades se realizan bajo la estructura de pequeño grupo y, en menor 
medida, de gran grupo, esto no significa la anulación del trabajo individual. No obstante, lo que sí se sustituye es el trabajo individual en solitario por el trabajo individual dentro del equipo.

c) Colaboración Familia-Centro. Un primer paso importante para conseguir esta colaboración es que los padres y madres de nuestros alumnos se sientan informados. Para ello, les convocamos a una reunión en la que les explicamos lo que supone el desarrollo de esta Unidad Didáctica Integrada y la importancia de su ayuda y colaboración para el buen desarrollo de la misma.

d) Decisiones sobre evaluación. La evaluación se constituye en un elemento fundamental del proceso de enseñanza y aprendizaje. No sólo nos permite realizar un seguimiento (riguroso y sistemático) sino que, además, nos proporciona toda una serie de información, sumamente útil, a la hora de ir introduciendo las modificaciones y ajustes necesarios entre lo planificado y su desarrollo en la práctica.

\section{Fase II.: Presentación de la Unidad Didáctica Integrada al grupo clase y diseño definitivo de la UDI}

Diseñada la UDI nos situamos en la segunda fase del proceso general. Ésta se inicia cuando el grupo de profesores efectúa la presentación de lo diseñado a su clase. Dicha presentación encierra una triple finalidad. Por una parte, pretendemos que los alumnos obtengan una visión global del trabajo que van a desarrollar y lo que implica esta propuesta desde los supuestos de la Integración Curricular. Para ello, utilizamos los mapas de conceptos elaborados, los cuales se constituyen en el eje central de la presentación que vendrá acompañada de la presencia de todo el profesorado implicado en el diseño y desarrollo de la unidad. Por otra parte, se incluyen en esta presentación una serie de contenidos de la UDI, los cuales deben poseer un marcado carácter motivacional. Se trata de que los alumnos, a través de una serie de actividades, obtengan la necesaria motivación para iniciar con interés y emoción la unidad, de forma que puedan conocer, de forma anticipada, lo que van a realizar, cuándo, en qué espacios, con qué materiales, qué salidas, con qué organización, cómo se va a evaluar, etc. Este momento de presentación se aprovecha para realizar una radiografía de los conocimientos que los alumnos poseen respecto al tópico. El cierre de esta presentación (a la cual se dedica no más de dos jornadas escolares) se constituye en el espacio idóneo para redefinir lo diseñado a fin de adaptarlo al grupo-clase. Es conveniente efectuar grabaciones 
en vídeo y tomar notas de campo sobre distintos aspectos de la presentación que, previamente, habrán sido identificados como prioritarios, con el fin de evaluarlos en un momento ulterior por el grupo docente. La información, que hayamos recopilado durante la presentación de la UDI a nuestros alumnos, se constituye en el material nuclear alrededor del cual habremos de ponernos a trabajar. Se abre entonces un espacio de trabajo intenso entre el grupo de profesores. Es el momento de los ajustes entre lo diseñado y lo que el grupo sabe, debe y desea saber sobre el tópico; es el momento de diseñar nuevos materiales, o bien de adaptar los ya elaborados; de tomar decisiones sobre la composición de los grupos de trabajo y un largo etcétera. El resultado final de esta tarea es el diseño definitivo de la UDI, que se desarrollará en torno a un Proyecto de Trabajo.

\section{Fase III.: El Proyecto de Trabajo. Análisis, síntesis y transferencia del conocimiento}

La tercera fase del proceso (denominada "El proyecto de trabajo. Análisis, síntesis y transferencia del conocimiento") se centra en la puesta en marcha de la unidad. Ésta puede durar entre un mínimo de dos semanas y un máximo de seis. El proceso de enseñanza-aprendizaje se organiza alrededor de un proyecto de trabajo que discurre en torno a dos momentos diferentes, no sólo por el tiempo dedicado a cada uno de ellos, sino por los objetivos y tipos de tareas que tienen lugar.

Así, el periodo dedicado al análisis y síntesis del conocimiento ocupa casi la totalidad del tiempo dedicado a la UDI. En dicho periodo los alumnos:

- Manejan el conocimiento que aparece integrado en una serie de núcleos de contenido, operando dentro de las distintas actividades propuestas;

- Obtienen información de distintas fuentes (documentación de clase, biblioteca del centro y del barrio, familia, Internet, etc.);

- Elaboran la información dentro de una propuesta metodológica, en la que cada contenido aprendido apoya la adquisición del siguiente, haciéndose materialmente visible la construcción del aprendizaje.

Este proceso culmina con un tiempo (entre una y dos jornadas escolares) dedicado a la transferencia del conocimiento. Transferencia entendida no (única y exclusivamente) como fase terminal y con entidad propia, sino como una capacidad que profesores y alumnos han de desarrollar a medida que transitan desde la planificación hasta la acción educativa. Para nosotros: 
[...] la transferencia del conocimiento es algo consustancial a la construcción de unidades didácticas integradas, desde el momento en que uno de los rasgos fundamentales que la caracteriza es su capacidad para transferir el conocimiento. Nótese que la propia integración curricular implica que los contenidos de cada una de las parcelas del conocimiento se interrelacionen de manera natural, entre sí y en su totalidad. Por tanto, la transferencia está presente no sólo en el trabajo a desarrollar con los alumnos, sino desde el momento en que los profesores inician la tarea de planificar la UDI. Además, la transferencia del conocimiento supone no sólo poder contar con la necesaria movilidad y adecuación de los contenidos, entre e inter-áreas de conocimiento, sino también entender que la transferencia debe poder capacitar al alumno para utilizar lo aprendido en contextos diferentes y distintos al educativo. Por último, la transferencia supone, además, funcionalidad, desde el momento en que si ambos conceptos ofrecen unidad, estaremos en el camino de conseguir que los alumnos comprendan y experimenten que el conocimiento no sólo se relaciona entre sí, sino que es útil y valioso para solucionar problemas de la vida cotidiana, ajustar mejor sus expectativas, etc. En definitiva, para comprender y situarse mejor en el contexto socio-cultural en el que les ha tocado vivir (ILLÁN; PÉREZ, 1999, p. 87-91).

\section{Fase IV.: La Evaluación. Fuente de aprendizaje y mejora}

La evaluación se constituye en la última fase de nuestro modelo procesual de diseño y desarrollo de Unidades Didácticas Integradas. Evaluación entendida, no como un trámite burocrático y de obligado cumplimiento, sino como una ocasión para que profesores y alumnos compartan escenarios a la hora de analizar y reflexionar en torno al proceso de innovación que han desarrollado (TÓJAR; MATAS, 2005). Se trata, por tanto, de crear las condiciones necesarias para que el plan de evaluación se constituya en un espacio de formación, al igual que lo fue el diseño de la UDI, a través del cual puedan llegar a identificarse aquellos elementos y cuestiones que precisan ser modificadas, con la finalidad de mejorar el proceso llevado a cabo y poder así incluir la UDI en el Proyecto Curricular del centro. Nos situamos, pues, ante una propuesta de evaluación que se va construyendo por todos los implicados, los cuales negocian y deciden qué evaluar, cómo y bajo qué condiciones. El hecho de contemplar una cuarta fase, donde la evaluación ocupa el último escalón de nuestro modelo, no significa que ésta no ha de ser contemplada en el transcurso de las tres fases precedentes. Así, cuando un grupo de profesores inicia el diseño de la UDI, no sólo toma 
decisiones respecto a qué objetivos responde el tópico, qué contenidos y qué actividades, sino que se toman decisiones sobre el modo en que va a ser evaluado, tanto el proceso de enseñanza-aprendizaje como el propio desarrollo de la UDI en su conjunto. En definitiva, las decisiones y acciones sobre evaluación han de impregnar todo el diseño y desarrollo de la Unidad Didáctica Integrada, aunque decidamos dedicar un espacio a la misma, el cual podríamos calificar como la evaluación de la evaluación. Como señala:

En este sentido, someter el informe - y el diseño, proceso y resultados de la propia evaluación - a la consideración de todos los implicados y discutir su contenido o contrastarlo con las interpretaciones que hagan de los resultados, son opciones válidas para dar credibilidad a su utilización en la toma de decisiones, así como para ir determinando progresivamente los criterios a utilizar en sucesivas experiencias. Pensemos que la finalidad de juzgar la calidad de la evaluación es la de detectar cualquier tipo de arbitrariedad que se haya podido cometer, así como estimar su alcance; no sólo en relación a errores técnicos, sino también en cuanto a interpretaciones excesivamente simplistas o equívocas de los resultados. Todo ello, en definitiva, implica una excelente oportunidad para ir aprendiendo a "hacer escuela" en colaboración y para participar en una verdadera experiencia social que va más allá de la mera planificación, puesta en práctica y evaluación de una unidad didáctica integrada (NIETO, 1999, p. 96).

Dicho esto abordamos, en primer lugar, bajo qué grandes presupuestos se sitúa nuestro modelo a la hora de plantearnos la evaluación del proceso de enseñanza-aprendizaje (sobre este particular ya hemos efectuado algunos comentarios al describir la Fase I) y, en segundo lugar, qué estrategias pueden resultar útiles a la hora de trazar un plan de evaluación del proceso de mejora llevado a cabo con motivo del diseño y puesta en marcha de la UDI. En ambos casos, basaremos nuestra exposición en los materiales que, sobre el particular, ha ido generando el Proyecto Atlántida en el que hemos participado. Nótese que se trata de dos procesos diferentes, que se llevan a cabo en momentos también diferentes pero que, dado el carácter dinámico y holístico de nuestro modelo, han de ir construyéndose y reconstruyéndose, en constante interacción el uno con el otro. En definitiva, no podemos desligar la evaluación del proceso de enseñanza-aprendizaje de la evaluación del proceso de mejora. Hacerlo, nos haría entrar en fuertes contradicciones, desde el momento en que lo que el alumno hace, aprende y siente, no puede ser desligado de la propuesta metodológica, 
de los materiales, de los espacios, del clima relacional que el diseño de la UDI propone, de su relación con el maestro, del ambiente de aprendizaje en el que tiene lugar la adquisición del conocimiento, ni tampoco del propio plan de acción que el grupo de profesores ha desarrollado.

\section{La evaluación del proceso de enseñanza-aprendizaje}

Nos situamos ante una opción en la que la evaluación no es algo finalista, ni tampoco un instrumento de control, sino que ésta, al igual que el resto de los elementos del currículo, ha de formar parte esencial del proceso de enseñanza-aprendizaje. Lograr una adecuada integración de la evaluación en el desarrollo del currículo, teniendo siempre como referencia el modelo de diseño en el que nos situamos y los supuestos que guían una educación democrática, implica tomar en consideración los siguientes principios básicos (PROYECTO ATLÁNTIDA, 1998, p. 41):

- La evaluación debe centrarse en la mejora más que en el control;

- La evaluación debe estar más atenta a los procesos que a los productos;

- La evaluación debe ser lo más integral posible;

- La evaluación debe realizarse teniendo en cuenta las situaciones individuales y grupales, así como los contextos institucionales y socioculturales;

- La evaluación es un proceso en el que deben participar todos los implicados.

Si efectuamos un recorrido por las tres primeras fase de nuestro modelo, veremos cómo cada uno de estos principios se desprenden de forma natural. Así, el trabajo colaborativo del profesorado en torno al diseño de la UDI (Fase I) crea las condiciones y oportunidades necesarias para que se lleve a cabo una construcción compartida, no sólo para llegar a acuerdos sobre lo que el alumno debe aprender (contenidos integrados), sino para identificar y consensuar los criterios valorativos de ese aprendizaje. En definitiva, los profesores, al poseer una visión global del proceso de enseñanza-aprendizaje a desarrollar a través del Proyecto de Trabajo (Fase III) (en lugar de una visión atomizada por áreas), llegan a acuerdos sobre los criterios generales de evaluación y en qué medida cada disciplina puede contribuir a la construcción de una evaluación integral $\mathrm{o}$, como se dice en estos días, a la adquisición de determinadas competencias. Por otra parte, la consideración de la diversidad como un valor positivo y en 
su sentido amplio (no restringido, que sería aquel que identificaría diversidad con discapacidad, dificultad, diferencia, etc.) está presente a lo largo de todo el proceso en torno al cual se desarrolla nuestro modelo. Cuando negociamos con nuestros alumnos el tópico elegido, ya contemplamos no sólo lo que el grupo-clase sabe respecto a un tópico determinado, sino que identificamos la posición individual de cada niño ante el tema propuesto. En consonancia con este planteamiento, la selección de los contenidos y el diseño de las distintas actividades integradas incorporan tanto lo que ha de aprenderse, como también cuándo, cómo, a través de que recursos y considerando los diferentes ritmos de aprendizaje. Así, la identificación de esos criterios generales de evaluación llevaría consigo su adaptación, no sólo a las características individuales, sino también a las grupales y contextuales previamente al inicio de la UDI. La utilización de criterios comunes para la evaluación,

[...] además de que ejerce un control externo sobre el currículum que desarrollan los centros y presupone una uniformidad en el modo de organizar, enseñar y evaluar dicho currículum que ni es real ni es deseable, puede provocar situaciones muy injustas sobre las que el alumnado no tiene ninguna responsabilidad (PROYECTO ATLÁNTIDA, 1998, p. 33).

Como podrá entenderse, la utilización de criterios comunes para la evaluación no encuentra sustento en nuestro modelo, al tiempo que se da de bruces con el entendimiento amplio de lo que supone la diversidad humana. Por último, y en consonancia con los argumentos expuestos hasta el momento, la evaluación del proceso de enseñanza-aprendizaje ha de ser consecuencia de un proceso de construcción conjunta y de un clima de confianza entre profesores y alumnos.

Esto no tiene nada que ver con dejación de responsabilidades, ni con la promoción de una cultura del laisez-fair, ni con nada que se le parezca. Se refiere a la necesidad de construir participativamente las reglas, de distribuir responsabilidades de común acuerdo, de exigir el cumplimiento de dichas responsabilidades con energía y rigor (PROYECTO ATLÁNTIDA, 1998, p. 44).

Indudablemente, la participación del alumnado en su evaluación significa un mayor compromiso e implicación, una mayor capacidad de autocrítica y 
autorreflexión, y una mejor comprensión de lo que se aprende y del cómo se aprende. Esto es lo que buscamos al presentar la Unidad Didáctica Integrada al grupo-clase en el transcurso de nuestra segunda fase, en la que efectuamos una presentación del diseño de la unidad, a partir de la cual efectuaremos las modificaciones y los ajustes necesarios.

\section{La evaluación del proceso de mejora}

Por último, y formando parte de la descripción de la cuarta fase de nuestro modelo procesual de diseño y desarrollo de unidades didácticas integradas, abordaremos en este apartado aquellos aspectos que tienen que ver con la evaluación del proceso de mejora. En el transcurso de las tres fases anteriores, ya hemos puesto de manifiesto que el trabajo del profesorado, en torno al diseño, desarrollo y evaluación de unidades didácticas integradas, se constituye en el motivo a partir del cual un grupo de profesores decide iniciar un proceso de mejora. Es decir, desde nuestro modelo entendemos que cualquier iniciativa debe enmarcarse dentro de un plan de acción, el cual debe poder ser evaluado a fin de analizar el camino efectuado y poder así encontrar aquellos indicadores que contribuyan a la solución de las dificultades identificadas. En definitiva, si se pretende que el trabajo del profesorado transcienda más allá de los límites impuestos por la puesta en marcha de una determinada innovación, ésta ha de poder ser documentada y constituirse, al tiempo, en un material valioso para la reflexión dentro de un espacio eminentemente formativo. Desde esta perspectiva, junto a la evaluación del proceso de enseñanza-aprendizaje, habremos de identificar qué elementos del proceso deben ser sometidos a evaluación, en qué momento del proceso y a través de qué procedimientos. Este tipo de decisiones, las cuales ya se van perfilando en la primera fase, encuentran su desarrollo en el transcurso de todo el proceso. Se trata de un tipo de decisiones, no generalizables a otros contextos y situaciones, dado que deben encontrar significatividad en el marco en que se desarrolla una determinada propuesta de innovación y cambio.

Por fin, decir que de los tres tipos de evaluación característicos de los procesos de cambio y de mejora (evaluación de la mejora, evaluación para la mejora y evaluación como mejora), descritos en los documentos del Proyecto Atlántida (2000, p. 11), nosotros nos decantamos por la comprensión de la evaluación entendida como mejora. Así, la evaluación como mejora significaría que ésta pasa a formar parte de la cultura del centro y es entendida como una tarea que potencia el cambio de la escuela por parte de sus integrantes. Es decir, se 
concibe como una forma de ejercer el autocontrol de lo que se está haciendo. En definitiva, cuando un grupo de profesores se sitúa bajo los supuestos de este tipo de evaluación, inicia un proceso de aprendizaje a través del cual se dota de las estrategias necesarias para poder llevar a cabo la autoevaluación, con el fin de poder disponer de los argumentos necesarios que expliquen y justifiquen su actuación ante su propia comunidad y ante cualquier evaluación externa a la que sea sometido. En suma, "supondría que la escuela asume que la evaluación es algo positivo y útil para su funcionamiento democrático y eficaz, abandonando las concepciones amenazantes y burocráticas que han prevalecido hasta ahora" (PROYECTO ATLÁNTIDA, 2000, p. 11). Llegados a este punto, nos corresponde cerrar este trabajo. Sintetizar todo este proceso en unas páginas no ha sido una tarea fácil pero, sin lugar a dudas, nos ha resultado tan emocionante como haber estado junto a todos esos niños, jóvenes y profesores que, durante más de una década, nos han permitido hacer posible nuestro sueño de ver crecer juntos, en un mismo escenario, a personas diferentes, con capacidades distintas, aprendiendo conjuntamente con felicidad y de un modo contagiosamente emocionante. Ojalá que estas letras sirvan para que el lector, que hoy nos acoge, dirija su mirada hacia nuestras huellas y nos ayude a seguir construyendo mejores escuelas, mejores aulas, mejores centros y mejores prácticas con las que impere la alegría, la felicidad y la emoción por el conocimiento; aquella emoción que únicamente percibe la piel de quien escuchó al niño llorar y sonreír a un mismo tiempo.

\section{REFERENCIAS}

ADELMAN, N. E. Te case for integrating academic and vocational education. Washington: National Assessment of Vocational Education, 1989.

ALMEIDA, L. S.; GUISANDE, M. A.; PRIMI, R.; LEMOS, G. La contribución del factor general y de los factores específicos en la relación entre inteligencia y rendimiento escolar. European Journal of Education and Psychology, v. 1, n. 3, p. 49-59, 2008.

ARNÁIZ, P.; LOZANO, J. Proyecto curricular para la diversidad. Psicomotricidad y lectoescritura. Madrid: CCS, 1996.

COLL, C. Concepción constructivista y planteamiento curricular. Cuadernos de Pedagogía, n. 188, p. 8-11, 1990.

. Psicología y currículum. Barcelona: Laia, 1987. 
COLL, C. et al. Actividad conjunta y habla: una aproximación al estudio de los mecanismos de influencia educativa. Infancia y aprendizaje, n. 59-60, p. 189-162, 1992.

ECHEITA, G.; MARTÍN, E. Interacción social y aprendizaje. En: COLL, C.; PALACIOS, J.; MARCHESI, A. (Comp.). Desarrollo psicológico y educación. Madrid: Alianza, 1990. v. III. p. 49-67.

GÓMEZ-GRANELL, C.; COLL, C. De qué hablamos cuando hablamos de constructivismo. Cuadernos de Pedagogía, n. 221, p. 8-10, 1994.

ILLÁN, N.; PÉREZ, F. La construcción del Proyecto Curricular en la Educación Secundaria Obligatoria. Opción integradora ante una sociedad intercultural. Málaga: Aljibe, 1999.

LOZANO, J.; ILLÁN, N. El euro para todos. Una experiencia pedagógica. Madrid: CCS, 2001.

MARTÍN-KNIEP, G.; FEIGE, D.; SOODAK, L. Curriculum integration: an expanded view of an abused idea. Journal of Curriculum and Supervision, v. 10, n. 3, p. 227-538, 1995.

MEC. Proyecto curricular de Educación Primaria. Madrid: MEC, 1992.

MOLINA, J. Will be specials the educational needs of students?: The intentional speech. Revista Lusófona de Educação, n. 13, p. 155-170, 2009.

MOLINA, J.; ILLÁN, N. Educar para la diversidad en la escuela actual: una experiencia práctica de integración curricular. Sevilla: MAD Eduforma, 2008.

NIETO, J. M. La experiencia de la unidad integrada desde la perspectiva de los alumnos y profesores. En: ILLÁN, N.; PÉREZ, F. La construcción del proyecto curricular en la educación obligatoria. Opción integradora ante una sociedad intercultural. Málaga: Aljibe, 1999.

POZO, J. I. Teorías cognitivas del aprendizaje. Madrid: Morata, 1989.

PROYECTO ATLÁNTIDA. Proyecto curricular. Escuela y valores democráticos. Madrid: F.E. CCOO, 1998.

. Proyecto de trabajo para facilitar los procesos de mejora en los centros comprometidos con (re)construcción democrática. Madrid: F.E. CCOO, 2000.

PUJOLÀS, P.; RUIZ, R. Atención a la diversidad, enseñanza personalizada y aprendizaje cooperativo. En: CONGRESO INTERNACIONAL DE ATENCIÓN A LA DIVERSIDAD, 2., 2000, Murcia. Anales... Murcia: Universidad de Murcia, 2000.

RELAN, A.; KIMPSTON, R. Curriculum integration: a critical analysis of practical and conceptual issues. Paper presentde at the Annual meeting of the American Educational Research and Improvement, 1991.

SOLÉ, I. ¿Se puede enseñar lo que se ha de construir? Cuadernos de Pedagogía, n. 188, p. 33-35, 1991. 
TÓJAR, J. C.; MATAS, A. El proceso de innovación educativa en la formación permanente del profesorado universitario: un estudio multicaso. Revista Española de Pedagogía, v. LXIII, n. 232, p. 529-552, 2005.

TORRES, J. Globalización e interdisciplinariedad: el curriculum integrado. Madrid: Morata, 1994.

VYGOSTKY, L. S. Pensamiento y lenguaje. Buenos Aires: La Pléyade, 1979.

ZABALA, A. El enfoque globalizador. Cuadernos de Pedagogía, n. 168, p. 22-27, 1989.

Texto recebido em 09 de dezembro de 2010.

Texto aprovado em 02 de março de 2011. 